\title{
Special considerations when planning experiments in a continuous process
}

\author{
ERIK VANHATALO AND BJARNE BERGQUIST \\ Quality and Environmental Management \\ Luleå University of Technology, Luleå, Sweden \\ Erik.Vanhatalo@ltu.se; Bjarne.Bergquist@ltu.se
}

\begin{abstract}
Discontinuous processes dominate experimental applications in practice as well as in literature. Continuous processes constitute a significant part of goods production, and the need to gain knowledge using experiments are as relevant in such environments as in e.g. parts production. We argue that the characteristics of continuous processes affect the prerequisites for experimental efforts to such an extent that they need special attention. To describe considerations when planning experiments in a continuous process, experiments performed in a blast furnace process are studied. We propose a tentative list of special considerations, which are discussed and summarized in a thirteen-step check list.
\end{abstract}

Keywords: Experimental design; industrial experimentation; engineering judgements, design of experiments, process control.

\section{INTRODUCTION}

Industrial processes are frequently discontinuous, such as when parts or batches are produced, e.g. robot welding of car bodies, heat treatment of springs and so forth, and the literature is filled with examples of full or fractional factorials as well as response surface experiments taken from these types of processes. From an experimental viewpoint, it is advantageous to have discrete units to work with, since changes of treatments may be measured on a particular piece. Accordingly, discontinuous processes dominate Design of Experiment (DoE) applications in practice as well as in literature. Nonetheless, the need for experimentation is not limited to discontinuous processes; see e.g. Wormbs et al. (2004) or Eriksson et al. (2006). Hence, this paper will discuss experimental considerations with special emphasis on the planning phase in another key category of processes - the continuous ones.

Continuous processes together with batch processes are frequent in process industry and use nondiscrete materials (Dennis \& Meredith, 2000). In continuous processes the product gradually and with minimal interruptions passes through a series of different operations and exhibits characteristics such as liquids, powders, and slurries (ibid). Typically, process control is a necessity due to varying properties of incoming goods. The processing steps include procedures where the product assumes shapes or forms where it is neither practical nor possible to track a single unit, such as when the product is in liquid, pulp, powder, slurry state or in granulate form. In addition, the product generally passes through pumps, chutes and conveyor belts, sometimes involving reverse flows of fractions of the product stream. Traceability is therefore complex, and on a detailed level practically impossible. Inevitably, the characteristics of continuous processes demand special consideration from the experimenter compared to running experiments in a discrete process setting.

For that reason the purpose of this paper is to describe some of the special considerations that the experimenter faces when planning experiments in a continuous process environment.

\section{PLANNING A DESIGNED EXPERIMENT}

An experiment can be separated into three phases: pre-experimental planning, performing the experiment, and post-experimental analysis. In this paper we focus on the considerations and 
problems that need to be tackled during the first of these phases. These are however inescapably affected by complications occurring when performing and analyzing the experiment as well. To help experimenters, guidelines for planning designed experiments can be found in literature. However, we have found no prior work that has focused on considerations for experimental planning in continuous processes. Yet, the guidelines found in literature are typically of a general nature and may thus serve as a starting point also for experimental planning in continuous processes. In Table 1, a compilation of important steps in the experimental planning process found in DoE literature is presented.

Table 1. Twelve important steps in the experimental planning process. The order of these steps varies somewhat between authors. An "X" in the table indicates that the step is explicitly stated. An "X" put in brackets indicates that the step is implied or vaguely described.

\begin{tabular}{|c|c|c|c|c|}
\hline Steps in the planning process & $\begin{array}{c}\text { Phadke } \\
\text { (1989) }\end{array}$ & $\begin{array}{l}\text { Coleman } \\
\quad \& \\
\text { Montgomery } \\
(1993)\end{array}$ & $\begin{array}{l}\text { Schmidt } \\
\quad \& \\
\text { Launsby } \\
(1994)\end{array}$ & $\begin{array}{l}\text { Dean } \\
\& \\
\text { Voss } \\
(1999)\end{array}$ \\
\hline $\begin{array}{l}\text { 1. Formulate the problem and define the objectives of the } \\
\text { experiment. }\end{array}$ & $(\mathrm{X})$ & $\mathrm{X}$ & $\mathrm{X}$ & $\mathrm{X}$ \\
\hline 2. Collect relevant background information. & $(\mathrm{X})$ & $\mathrm{X}$ & $(\mathrm{X})$ & \\
\hline 3. Choose response variable(s) & $X$ & $\mathrm{X}$ & $\mathrm{X}$ & $\mathrm{X}$ \\
\hline $\begin{array}{l}\text { 4. Identify control variables, held constant factors and their } \\
\text { levels as well as disturbance factors. }\end{array}$ & $\mathrm{X}$ & $\mathrm{X}$ & $\mathrm{X}$ & $\mathrm{X}$ \\
\hline $\begin{array}{l}\text { 5. If possible, specify normal levels, measurement precision, } \\
\text { proposed settings or desired levels and related strategies, and } \\
\text { predicted effects of control variables and held constant and } \\
\text { disturbance factors. }\end{array}$ & & $\mathrm{X}$ & $\mathrm{X}$ & \\
\hline 6. List known and suspected factor interactions. & & $\mathrm{X}$ & $\mathrm{X}$ & $(\mathrm{X})$ \\
\hline $\begin{array}{l}\text { 7. Choose experimental design and experimental procedure. } \\
\text { This includes activities such as choosing sample size and } \\
\text { analyzing pre-experimental power. }\end{array}$ & $(\mathrm{X})$ & $\mathrm{X}$ & $(\mathrm{X})$ & $\mathrm{X}$ \\
\hline $\begin{array}{l}\text { 8. Consider restrictions and anticipated difficulties in running } \\
\text { the experiment. }\end{array}$ & & $\mathrm{X}$ & $(\mathrm{X})$ & $\mathrm{X}$ \\
\hline 9. Outline the analysis techniques. & $\mathrm{X}$ & $\mathrm{X}$ & $\mathrm{X}$ & $\mathrm{X}$ \\
\hline 10. Assign responsibility for coordination of the experiment. & & $\mathrm{X}$ & $(\mathrm{X})$ & \\
\hline 11. Perform a trial run or pilot experiment if possible. & & $\mathrm{X}$ & & $\mathrm{X}$ \\
\hline 12. Review and revise the above steps if necessary. & & $\mathrm{X}$ & & $\mathrm{X}$ \\
\hline
\end{tabular}

From Table 1, it is clear that the most detailed checklist is given in the seminal paper by Coleman \& Montgomery (1993), while Phadke (1989) can be viewed as more synoptic. We have tried to locate more recent literature but have not found work that significantly adds to the twelve steps in Table 1. Even though the authors in Table 1 do not stress the same steps of the planning process exactly, there seems to be a reasonable agreement of which activities that are held important for the experimental planning process.

\section{METHOD}

There are several ways to generate knowledge on how to work with experiments in continuous processes, but due to the purpose of describing the many specific issues that experimenters face, it was decided that a single case study constitutes a good choice due to the possibility of following the work of engineers for an extended time period. In this work, an experimental blast furnace (EBF) operation was selected as study object since an initial discussion with the plant engineers revealed that they were interested in DoE and factorial experiments. Also, planning, performing and analyzing factorial type experiments in this setting was considered to provide a basis for interesting comparison with the prevailing experimental practice at the site. However, they lacked the statistical know-how to implement the technique on their own, and it was decided that the authors of this paper could aid the experimental work. As such, this study also includes elements of action research; see e.g. 
Lewin (1947). Another benefit was that one of the authors had previous experiences with full scale and pilot scale experimentation in continuous and batch processes in a metallurgical environment (Bergquist, 1997, 1999).

\subsection{The case: an experimental blast furnace}

Today, the blast furnace steelmaking process is the primary source of the world's steel production. The blast furnace can be characterized as a high temperature counter current reactor for reduction and smelting of iron ore into hot metal (Geerdes et al., 2000). Coke and coal are used to reduce iron oxide, normally in form of sinter and/or pellets, into liquid iron.

In 1997, Luossavaara-Kiirunavaara AB (LKAB), a leading Swedish producer of highly developed iron ore products, pellets in particular, inaugurated a pilot scale blast furnace. Although the experimental cost per run and risks associated with performing experiments are great even in pilot scale, they are substantially lower than they would have been in full scale operation. The EBF is specifically designed for experimental purposes and has many possibilities for measurement during operation. An outline of the EBF, examples of measurement possibilities, and some specifications of the furnace are presented in Figure 1.

The general customer demands on iron ore pellets are that their blast furnaces may be run efficiently and effectively with few disturbances and that the resulting iron is of a high grade. Most experiments performed in the EBF therefore include response variables connected to the quality of the produced iron, such as chemical composition of iron and slag, as well as response variables related to energy efficiency and stability of the process itself, e.g. CO gas utilization.

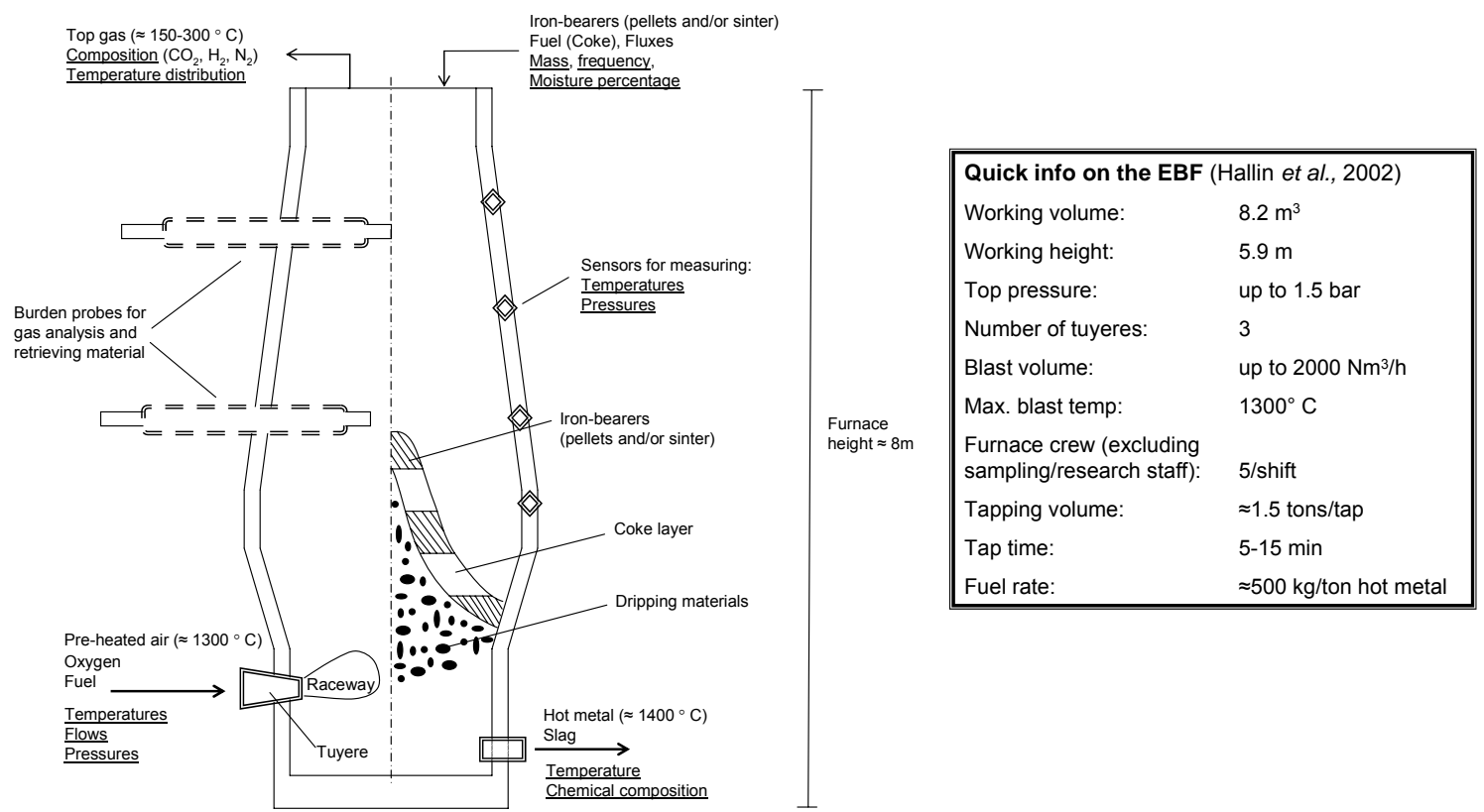

Figure 1. Outline of the Experimental Blast Furnace process inspired by Zuo (2000). In this outline a few examples of possible response variables are underlined.

\subsection{Data collection and analysis}

This research has been made in close collaboration with research engineers at the EBF plant (hereafter the "EBF engineers"). Within the case study, data has been collected using multiple sources: interviews; observations; discussions; as well as by the authors' active participation in planning, performing and analyzing experiments at the EBF plant during two experimental campaigns in 2006. This provided opportunity for data triangulation during analysis; see Yin (1994). At the start of the collaboration, in-depth semi structured interviews were conducted with five EBF engineers, with purpose to provide a "snapshot" of their prevailing methods for 
planning, performing and analyzing experiments prior to the intervention made by the authors. This snapshot is summarized in section 4 and should be contrasted to the authors' intervention which is described in section 5 .

\section{EXPERIMENTAL PROCESS PRIOR TO THE COLLABORATION}

In this section we synoptically describe the prevailing situation at the start of the collaboration. The experimental efforts at the EBF plant at this time were run in two experimental campaigns per year for about four to six weeks each time, and this had been the case since the plant was built. Several different experiments were usually run in each campaign and the experiments were typically divided into a number of "periods" (comparable to experimental runs). The experiments had many different objectives, e.g. product development, production development, customer trials, and external projects. The experimental efforts could be divided into three separate phases - planning for, performing, and evaluating the experiment. The planning process could further be divided into campaign planning (coordination of the different experiments in the campaign) and planning of the separate experiments. The focus of the collaboration project was on the latter. The existing experimental planning documentation was essential for the planning process and the responsible engineer(s) had the task of documentation of both the planning phase as well as of many of the events that occurred during the experiment.

The most frequent category of experimental factors was material factors, i.e. different types of iron bearers (mostly pellets). During most experiments, the process variables were held constant, making the material factor the only true experimental factor. However, in nearly all experiments, the coke rate was used to control the thermal state of the process during the experiment even though it was not viewed as an experimental factor. There were other experimental factors as well, e.g. charging method of the furnace and processing variables (such as the blast temperature), but these were studied in separate experiments. It was thus not possible to test material and process factor interactions.

Factorial type experiments were not common practice among the EBF engineers. In fact, many of the engineers were skeptical to factorial experiments because of the continuous nature of the process. Instead, the prevailing practice at the EBF had been to conduct a type of one-factor-at-a-time (OFAT) experiments, e.g. where one type of pellets was assigned as reference, and the others as experimental pellets. The experimental pellets' performance in the furnace was then evaluated in comparison to the reference pellets, where references were run between runs of experimental pellets; see Figure 2 for the general principle.

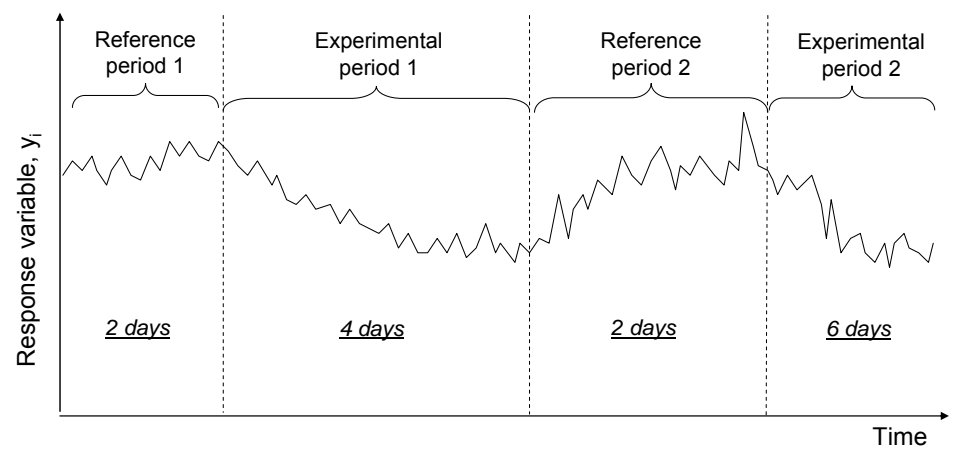

Figure 2. The general principle of one-factor-at-a-time experimentation at the EBF plant. The time indicated for each run in the figure are examples of a possible arrangement.

When performing the experiments, about ten LKAB personnel were usually present at the EBF plant during a twenty-four hour period. In addition, a furnace crew of five per shift was needed to run the EBF. Additional personnel were also required for maintenance and to run the logistics concerning inputs and outputs from the EBF. During operation, structured 
meetings were held between the engineering team and additional staff on regular occasions. Possible disturbances and other events were noted in logbooks as well as in meeting protocols and could thus be traced during analysis.

Analyzing experiments conducted in the EBF was a time consuming process and added experimental runs prolonged the analysis process. In Figure 3 the prevailing analysis process for experiments at the EBF is indicated.

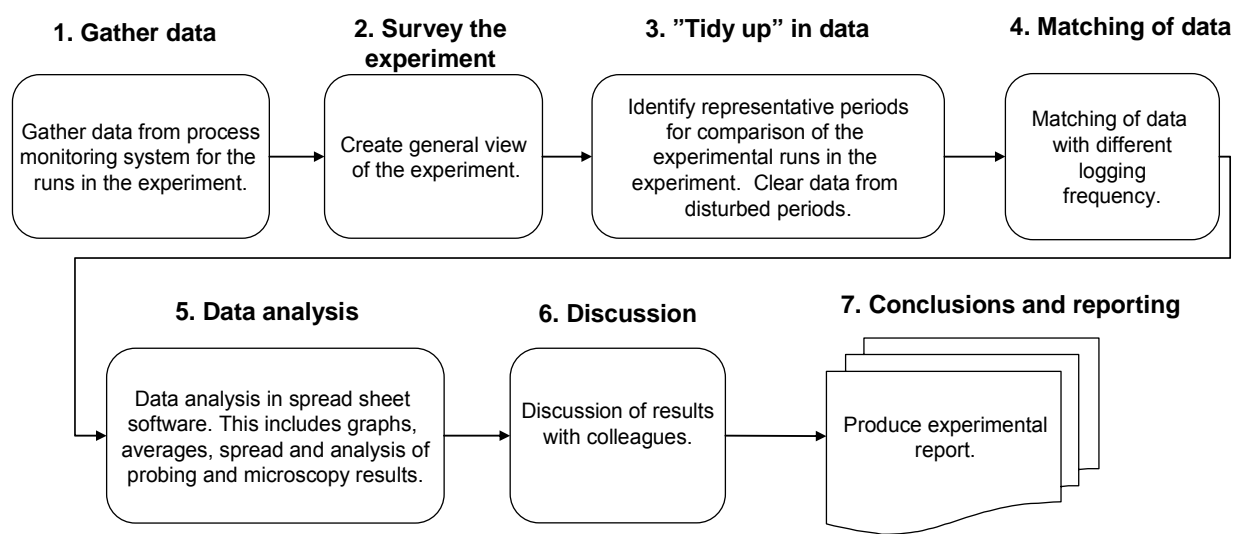

Figure 3. Main activities in the analysis process at the EBF plant at the start of the study.

Prior to the start of the study, the dominating data analysis tool used by EBF engineers was common spreadsheet software, with special emphasis on time-series plots over important response variables. Use of statistics software was not common practice among all, neither was multivariate analysis. However, several of the EBF engineers supported the development of multivariate analysis of experimental data.

\section{THE INTERVENTION - TESTING FACTORIAL DESIGNS IN THE EBF}

The intervention of the authors occurred after the current method of performing experiments at the EBF had been investigated and described. The main activities of the authors' research process are presented in Figure 4. Besides these, frequent discussions and meetings between the authors and EBF engineers have been held to present and discuss preliminary results.

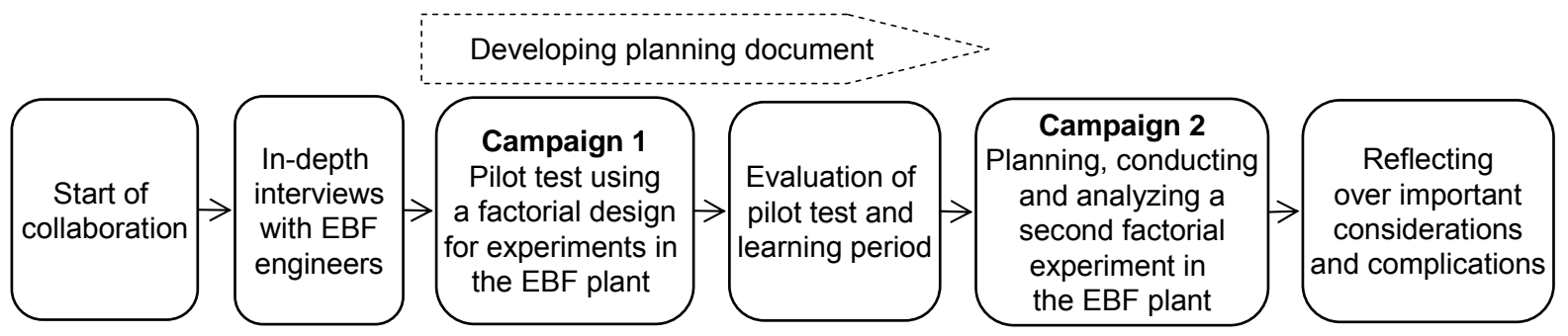

Figure 4. Summary of the main activities during the research process.

In campaign 1 (see Figure 4), a $2^{2}$ factorial design with center points was used to test the applicability of the factorial designs in the EBF. This can be viewed as a pilot test which generated both answers and new questions, e.g. what was the suitable length of the individual runs in factorial type experiments in the EBF? In campaign 2, a replicated $3 \times 2$ factorial experiment, testing three different materials together with a process factor was conducted; see Vanhatalo et al. (2007). Before, during and after Campaign 1, a planning document template was developed from existing documentation practice, incorporating recommendations foremost from Coleman and Montgomery (1993). This planning document was used during campaign 2 , where complications or special considerations during the planning process were noted by the authors. 
We have then reflected on and analyzed the experimental results, but also the observations made during the intervention in order to describe the considerations an experimenter faces in a continuous process; see section 6 . Finally, we have linked these special considerations to the steps of the planning process in Table 1 thereby summarizing our conclusions in section 7.

\section{LESSONS LEARNED FROM THE EBF CASE}

In this section we present and discuss a tentative inventory of special considerations and/or complications that need to be handled when planning experiments in continuous processes. These are developed from the studied case (the EBF setting) and discussed under the following sub-headings. The sequence in which the considerations are discussed in this section is not intended as a ranking of their importance.

\subsection{Large scale experimentation around the clock in a complex process}

Formulation of the problem(s) and defining the objective(s) are for good reasons the first step of any successful experimental effort, and this is true for experiments run in the EBF plant as well. The EBF process, as most types of continuous processes, has complex and expensive machinery, and the process is run for weeks or months without stops. Since running the EBF process day and night requires about thirty people (comparable to full scale production plants), information, communication, documentation and control is difficult but critical to experimental success. Also, running experiments around the clock restricts the level of involvement of the responsible engineer(s) in all process decisions made. We argue that especially in situations like the one described above, an important complication that emerges is to ensure that all who should be acquainted with the objectives are in fact informed.

Some of the disturbances that occur during the experiments may be foreseeable and for these events guidelines should be extracted and communicated to those involved, e.g. multiple working shifts. When other events occur, personnel at the plant must be made aware of the purpose of the experiment and how experimental conclusions may be drawn, so that actions taken do not interfere with the aim of the experiment. As one of the EBF engineers put it: "the experiments are in progress day and night, which makes it hard to ensure that all involved have all information. So of course, there are human disturbance factors too."

Lastly, a large portion of the time spent on planning experiments in the EBF can be assigned to logistics planning, e.g. fuel and raw material procurement, as well as the disposal of the produced iron. Even though it is a pilot scale operation, the handled volume of raw material and fuel is substantial. Logistics planning is therefore critical for experimental success in the EBF, as well as could be expected in other processes with similar characteristics.

\subsection{The need for process control - an important disturbance factor?}

An important experimental complication is that process control can be unavoidable in a continuous process environment. Without monitoring and controlling the reductant rate (hereafter the fuel ratio) during experiments in the EBF, there is a risk for the melt to freeze or overheat, which can jeopardize the experimental campaign, the plant as well as personal safety. The melt temperature control of the EBF is manual, requiring human deliberations at certain processing states, and the control includes a large but often unknown time lag. The great importance of the thermal state in the process in combination with the uncertainty of the manual control makes it an important disturbance factor. When the furnace runs low on fuel the time lag for adding extra fuel (coke) into the burden is a few hours, but the furnace may run on too lean burden mixes for a long time period, if previous runs were rich in fuel, leaving unconsumed coke in the reaction zone. For numerous experimental aims, however, the fuel ratio is also an important response variable, since fuel efficiency of the blast furnace process is an important customer demand. Whether the fuel ratio is a control variable, a disturbance factor or a response or all of the above have been the cause for intensive debate during the 
collaboration between the authors and the EBF engineers. It is however clear that the changes in fuel ratio will affect the process and it should therefore be treated as a covariate during analysis. The fuel ratio can thus be considered as a borderline between a response and a control variable. An alternative is to monitor the control variable instead of the primary response variable, but fuel ratio is still troublesome due to the subjective nature of the decisions regarding the amount of coke to add or subtract to maintain a proper thermal state. Further, if an experimental run produces a poor processing state, it might be necessary to abort the run or to add extra coke to avoid jeopardizing the process state for future runs in the experimental campaign. Another, but so far not tested option, viable under certain circumstances, would be to run the process with a constant coke rate and let the process temperature vary according to predefined control limits, or to use faster means of controlling the temperature such as direct injection of carbon powder into the reaction zone if the purpose of the experiment allows it.

Process monitoring and control of the EBF is further complicated due to time-delay in analyses of important variables that constitute basis for control, e.g. chemical quality of the produced iron. Since process control may be unavoidable during experimentation, it is vital that control strategies are established during the planning process of the experiment. We argue that this problem is important enough to justify "process control strategy" as an entirely separate step in the planning process for continuous processes. These strategies must be well documented and communicated to personnel involved in process control.

The selected strategy for controlling the process will affect many decisions during the planning process, e.g. the selection of which variables to hold constant during the experiment. Choosing to hold a variable constant may force the engineer to use another to control the processing state. Factors affect each other and the choice of factor levels becomes dependent on other factor levels. Thus, the choice of which factors to hold constant affects the possible control variables, and vice versa. In addition, e.g. material balance, energy balance and slag composition calculations are frequently necessary in order to make sure that the levels of experimental factors do not cause unwanted processing states.

\subsection{There are many possible disturbance factors in a complex process}

Complex processes imply many possibilities for disturbances or failures. Important disturbance factors in the EBF process are for example the moisture content of coke (fuel) and pellets, over or under compensation of fuel when controlling the process, and failure of process equipment. Controlling disturbance factors in continuous process experiments is a demanding task since each run may take several days. Also, the flows of raw materials may themselves be continuous and natural resources such as coal are wrought with the variation Mother Nature gives them. Events that disturb the flow of material in and out of the EBF furnace are generally considered as severe. The engineers at the EBF did not document possible disturbance factors and strategies to offset them in the planning phase at the start of the research project. This routine was however added when the planning document was further developed. Since using factorial designs was considered to restrict the possibilities for compensation of disturbances during the experiment; the pre-planning of strategies to neutralize the effects of disturbances became much more important.

In large, complex machine environments, break-downs of equipment become unwelcome, but often unavoidable ingredients of the experimental logbook. As such events may jeopardize the whole outcome of the effort, reliability and maintenance issues are critical, especially when experiments are run for longer time periods as is often the case when processes include buildup of pressures, temperature changes, phase changes etc. 


\subsection{The experimental unit, response variables and analytical considerations}

Selecting relevant response variables is crucial to achieve the objective of the experiment, i.e. to measure the experimental units' reaction to the treatments. However, defining the experimental unit for the experiments in the EBF is not straightforward. The complex physical flows in the process and the confined nature of the material flow in the process make determination of the experimental unit problematic. Instead of measurements taken on a unit having received a special treatment, the experimenter must find representative samples before and after any changes of treatments and measure effects based on the differences in these samples. In the EBF, no alternative was found to defining the experimental unit as samples of the process' performance taken during a pre-established time period of the production. To evaluate the performance, both output (iron and slag) characteristics as well as the processing state (process variables) include interesting response variables.

The blast furnace operation, like many process industry operations, has many inherent features that often prohibit direct measurement of interesting phenomena, such as most measurements inside the reaction zone. Therefore, process data are collected by measuring secondary process variables, e.g. shaft temperatures, pressure drops, and gas utilization. According to Hild et al. (2000) data from continuous processes are often dominated by frequent on-line logging of process variables while the measuring of product characteristics usually is made by off-line analysis, and limited and less frequent when obtainable. This description fits the EBF case well where iron and slag responses are recorded about once every hour. The difference in sampling frequency between on-line and off-line measurements is another obstacle (Hild et al., 2000). Matching of off-line responses and process variables becomes necessary for identification of cause-and-effect relationships between processing conditions and end-product characteristics. Also, using different groups of response variables, i.e. iron/slag data and measurements from the process, introduces another complication, namely time lagging of the different responses with respect to each other. Therefore, when these different groups of responses are analyzed together (e.g. when using multivariate techniques) time lags need to be considered, see Figure 5 for the general idea.

\begin{tabular}{|c|c|c|}
\hline Time & $\mathrm{Y}_{\text {process }}$ & $\mathrm{Y}_{\text {output }}$ \\
\hline 1 & $\mathrm{Y}_{\mathrm{p} 1}$ & $\mathrm{Y}_{\mathrm{o} 1}$ \\
\hline 2 & $\mathrm{Y}_{\mathrm{p} 2}$ & $\mathrm{Y}_{\mathrm{o} 2}$ \\
\hline 3 & $\mathrm{Y}_{\mathrm{p} 3}$ & $\mathrm{Y}_{\mathrm{o} 3}$ \\
\hline 4 & $\mathrm{Y}_{\mathrm{p} 4}$ & $\mathrm{Y}_{\mathrm{o} 4}$ \\
\hline 5 & $\mathrm{Y}_{\mathrm{p} 5}$ & $\mathrm{Y}_{\mathrm{o5}}$ \\
\hline 6 & $\mathrm{Y}_{\mathrm{p} 6}$ & $\mathrm{Y}_{\mathrm{o6}}$ \\
\hline$\ldots$ & $\ldots$ & $\ldots$ \\
\hline $\mathrm{n}$ & $\mathrm{Y}_{\mathrm{pn}}$ & $\mathrm{Y}_{\mathrm{on}}$ \\
\hline
\end{tabular}

Figure 5. Representation of time lag between processing response variables and output response variables. In this example a lag of 4 hours is assumed between reactions in process responses and the changes that can be expected in the output.

Choosing response variables in the EBF process is complicated due to their multitude. There are more than twenty responses extracted from the chemical analyses of iron and slag alone, and there are at least twice as many process variables, of which some could be interesting responses. Several of the response variables are correlated. In continuous processes, measured responses are typically not independent of one another, as a few underlying events often drive the process at any given time, and many responses may thus react to the same underlying event (Kourti \& MacGregor, 1995). One of many examples from the EBF case is that the amount of certain trace elements in the iron and slag is correlated to the temperature of the melt. Shifts in the processing state may be visible in multivariate representations but may, due to normal variation, not deviate significantly in univariate plots (Hild et al., 2000). Therefore, 
examining such responses one at a time makes interpretation difficult (Kourti \& MacGregor, 1995). Multivariate techniques will therefore constitute important analysis tools for experiments in continuous processes; see Vanhatalo et al. (2007). Kourti (2005), Duchesne \& MacGregor (2000) and Wikström et al. (1998) provide further examples of multivariate control and monitoring applications for continuous and batch processes, e.g. principal component analysis and projections to latent structures by use of partial least squares.

The frequent logging of many of the responses also warrants a high degree of autocorrelation in the response data, which can be problematic for some methods of analysis. Many of the logged variables in the EBF are calculated from other variables, and are thus linearly dependent, causing singularity problems during matrix inversions and should be avoided due to risks for large calculation errors.

We argue that in order to effectively and efficiently analyze the results from a continuous process a multivariate approach is needed, even though it often requires training. Assessing univariate responses and calculating many univariate effects is an inferior option, as it does not provide the desired fundamental overview of the experimental results. Also, calculating many separate univariate effects (using the same individual significance level) increases the $\alpha$-risk.

Further, to not miss important responses that could answer research questions not yet posed, or to not miss unanticipated vital variables, the EBF engineers often postpone the selection of variables until the start of the analysis. However, in doing so, an important element of pre-experimental planning is lost. We argue that it is important to discuss if the existing response variables are relevant for the objective of the experiment. Also, by discussing relevant response variables during the planning process, an important part of the analysis is done beforehand. We recommend partitioning the response variables into manageable groups that could be expected to reflect certain events in the process, to reduce the analysis work.

\subsection{Working at a detailed level in the planning process can be impractical}

Coleman \& Montgomery (1993) and Schmidt \& Launsby (1994) propose that normal levels and measurement precision of all involved variables should be determined, and also that proposed settings or desired levels, and predicted effects of control variables, held constant factors, and disturbance factors should be set. This step in the planning process implied a deeper focus on details than had previously been the case among the EBF engineers. Held constant factors had previously been documented but documentation of measurement precision, predicted effects of control variables, held constant factors, and noise factors was not common practice. Even though many of the questions during this step could not be answered directly and had to be left for the time being, it provided information of the unknown, e.g. the measurement precision of many variables. Most unknown measurement errors were considered systematic, due to e.g. calibration errors, and would thus not affect experimental effect calculations as dramatically as lack of precision and repeatability. Still, the work in this step triggered new ideas for improvement to secure the validity of experimental results.

However, following the recommendations in literature was not always practical. Having many response variables for which effects should be predicted made it impossible to work at such detail. Hence, we recommend starting by describing predicted process reactions by words, and by focusing on the most important anticipated reactions. In this way, a-priori knowledge about suspected process responses is still utilized; see Bergquist \& Vanhatalo (2007).

Considering possible factor interactions in the planning process is important as it directly affects the choice of experimental design. However, if the planning process includes handling some sixty responses (as was the case at the EBF), this step is difficult. Yet, factor 
interactions should be given consideration. In the EBF project, the possibility of interaction effects were investigated by asking and discussing questions like "if we change factor $\mathrm{A}$ on different levels of factor B, can we expect different reactions from the process manifested in groups of responses that describe phenomena that we are interested in?" An option not used in this case, but likely useful, is mining data archives to discover and document possible correlation patterns.

\subsection{The dynamic characteristic of the EBF process}

The EBF process exhibits time-delay in process responses (inertia) when being exposed to changes, e.g. going from one experimental run to another. Black-Nembhard \& ValverdeVentura (2003) differentiates between a dynamic and responsive system. In a dynamic system a period of delay will occur between the time that factor $\mathrm{X}$ is changed and the time that this change is realized in the response $\mathrm{Y}$, whereas in a responsive system, this change in $\mathrm{Y}$ is immediate. The inertia in the EBF is also dependent on which factors are changed and differs among process variables and responses. Changes in carbon injection feed rate would influence the composition of the furnace exhaust gas immediately, while it may take hours until such change would affect the liquid metal composition. Thus, it is not feasible to measure the effects of applied treatments to the EBF instantaneously as the impact of a treatment often requires time to reach an equilibrium state, which is common for continuous processes (Saunders \& Eccleston, 1992). The time required for the process to react to a disturbance and obtain a new steady state needs to be considered when determining the time needed for each experimental run. These time lags also become important during analysis of experimental results.

The EBF engineers have usually tackled this problem by disregarding all data during the period in which the process is suspected not to have reached the new steady state. This is a robust way of handling the lag uncertainty, but when performing factorial experiments it becomes costly due to the need of multiple runs. Depending on the disturbance, as much as twenty-four hours of response data were ignored during the analysis. Also, in the EBF process, steady-state is a matter of definition. In fact, the blast furnace process is inherently unstable and non-stationary (Zuo, 2000), and although engineering control is used, statistical stability is not obtained. The prevailing way to assure quality of results has consequently been to conduct quite lengthy experimental runs in contrast to carrying out more frequent changes of the factor levels. The choice to discard time periods when the process is shifting between steady states is also one explanation to why shorter runs were infrequent. When uncertainties exist as to how long it would take to change from one steady state to another, many deliberate changes would seemingly lead to costly and unproductive periods of the experiment.

Therefore, the experimenter will need to assess and compensate for the time needed for the treatments to reach full impact, and time lags will differ between responses. In the EBF project, the authors studied the time needed for effects to stabilize after the treatment was applied by studying multivariate time-series plots of latent variables, e.g. the behavior of principal component scores over time, see Figure 6. After the transient time has been determined, we recommend that the process data for these periods is removed from the experimental data matrix prior to the continued multivariate analysis. Another possibility is to treat each response separately. In this way an individual time lag could be established for each response variable in the data matrix. However, this approach was not used in the EBF project.

\subsection{Considerations affecting experimental design and procedure}

When conducting experiments in process industry, it is often sound to consider the evolutionary operation (EVOP) procedure introduced by George Box (Box, 1957). However, in the EBF case this procedure was disqualified since one of its basic prerequisites, production over a reasonable extensive period of time (Hahn \& Dershowitz, 1974), was not 
Authors post print of paper published in Quality Engineering, 19(3), 155-167; DOI $10.1080 / 08982110701474100$

considered to be fulfilled. In the EBF the available time for each experiment seldom exceeds three weeks of production.

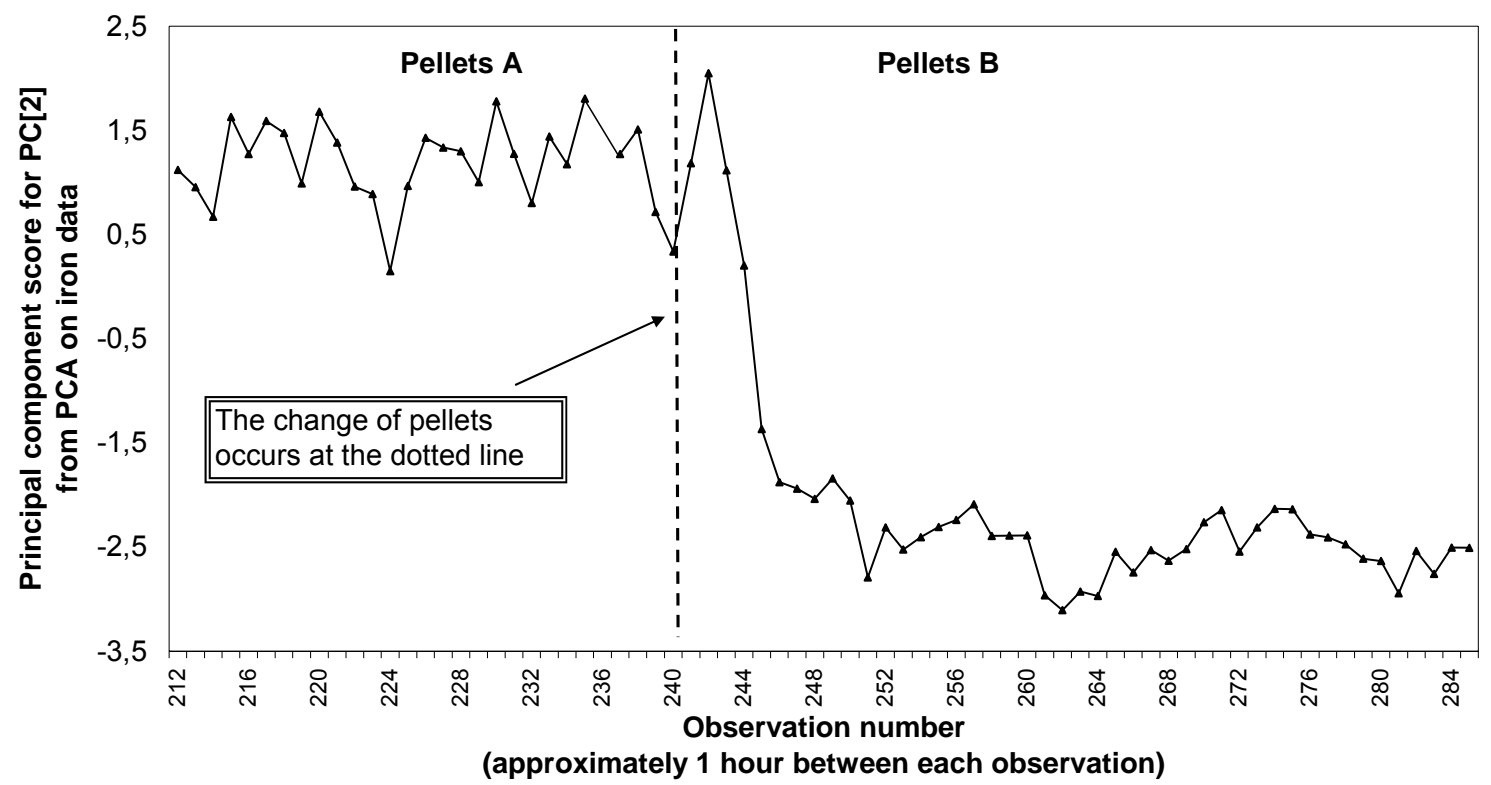

Figure 6. Time-series plot of principal component scores from a principal component analysis of iron data from campaign 1 in the EBF. The second principal component was considered to carry information connected to the different pellet types. This plot was used to determine the time needed for a shift in pellet type to reach full impact in the process, arguing that the iron responses would be the slowest to react. This plot indicates that at least 6 hours are needed before the effect in iron data stabilizes.

The prevailing OFAT strategy to experimentation at the EBF allows for an ad-hoc accentuated experimental work which has practical advantages since it allows the engineer to consider knowledge created during the realization of the experiment. Also, it leaves more freedom to react to incidents and problems encountered during the experiment compared to a factorial experiment. Hence, by using the OFAT type designs the EBF engineers have "gotten away with" a less detailed pre-experimental planning. However, there are serious drawbacks connected to the OFAT strategy such as a higher demand of resources, guesswork instead of precision when estimating the factor effects, lack of ability to estimate interaction effects and it provides experimental information in a smaller region of the factor space. We believe that it would be better that the EBF engineers did spend more time during pre-experimental planning and used other experimental arrays than what is reflected in Figure 2.

A problem that was highlighted by the use of factorial designs was the increased need for level changes, i.e. individual runs, compared to OFAT experiments. This, in combination with process inertia, was considered to require that experiments would have to run for longer time periods than the current OFAT type experiments. Thus, factorial designs were initially considered to be too costly. Therefore, it was necessary to find a way to minimize the time of transient periods between runs. Restricting the randomization of the runs by using the splitplot approach became necessary; see e.g. Federer \& King (2007) and Montgomery (2005). Since an important purpose of the EBF is to constitute a test-bed for product development of pellets, main effects of changed burden composition (pellets) are of utmost importance. Also, interactions between material factors and processing variables are highly interesting, whereas process variable effects are interesting only to create better models of how the EBF itself is working. Using a split-plot design where processing variables are assigned as whole-plot factors and the material factors as sub-plot factor provides good precision in estimating the material main effects and the interaction effects, while the cost of having lower precision of 
the process variables was a small price to pay compared to the option keeping them constant. A split-plot arrangement was also chosen for campaign 2, see Vanhatalo et al. (2007).

The need to restrict the randomization also arose due to the fact that trial runs were not possible in the EBF process due to a number of reasons: the blast furnace is only operated during two campaigns per year; each run is very expensive; and even if trial runs were possible the time available to learn from them would be too short before the real experiment must be performed. Performing trial runs must be considered sound and effective for revealing potential problems or misjudgments that one has made during the planning process and should be used whenever possible. Even though the accumulated organizational learning lessens the need for trial runs, since documentation and colleagues can be consulted there are still occasions where trial runs would be of help. Some factor combinations may, for example, be anticipated to invoke process difficulties during the experiment. If the results of the experiment are dependent on these runs (which often is the case), it may be advisable to start the experiment with these, even if that would interfere with a strictly randomized run order. In this way incompatible factor combinations may be detected at stages where alterations in the experimental design are feasible. Consequently if such runs produce unacceptable processing states, they could be aborted, the levels changed, and only one or two runs would have been lost. This approach can easily be combined with the split-plot design indicated above. However, any split-plot type design will lead to special considerations during analysis.

A specific aspect of running the EBF is that the interior of the furnace is subjected to buildup of crust of material or channels forming in the burden. Some of these process problems may be noticed during the experiment, while others go undetected. As a randomized run order often is impossible, split-plot designs should therefore be designed to prevent low frequency disturbances fogging results as much as possible, even if whole-plot effects are of less importance.

Another objection to factorial designs that the EBF engineers had was that long-term effects could not be evaluated as usual, i.e. such effects that could possibly appear after many days of operation in the furnace. We argue that factorial designs are not compatible with such experimental purposes if the extent of the experimental campaign is insufficient for even the smallest factorial, e.g. a $2^{2}$-experiment with a few replicated runs. On the other hand, if such long-term effects were to occur, the engineer would still have trouble to significantly assign this effect to the experimental factor if runs are not repeated.

A consequence of the complexity of the process is that important apparatus may start to malfunction during the experiment, or that the equipment is worn or deteriorating. If failures are considered as likely, the engineer running experiments for long time periods should consider designs that are not too dependant on each individual run, such as designs with run leverages less than 0.5 . Another possible strategy can be to leave some "unplanned" experimental time at the end of the campaign to accommodate for missing runs. Due to the slow nature of performing experiments in the EBF, relatively complex analysis may be performed during ongoing experiments. Therefore the engineer can decide to extend the time of individual runs if problems occur to achieve a reasonable period of stable processing conditions for each run. This strategy, called an "adaptive design" by Vanhatalo et al. (2007), was chosen and proved beneficial for the experiment in campaign 2.

\section{CONCLUSIONS AND DISCUSSION}

Running experiments in continuous processes implies several challenges in addition to the ones always present. In this section we sum up and discuss considerations that should be contemplated when planning an experiment in a continuous process. We start by presenting a summary of special considerations discussed in the previous section and linking them to and expanding the twelve-step planning process described earlier; see Table 2. 
Table 2. Summary of special considerations and their linkage to the steps of the planning process.

\begin{tabular}{ll}
\hline Steps in the planning process & Special considerations \\
\hline $\begin{array}{ll}\text { 1. Formulate the problem and define } & \bullet \text { All involved, including operators, need to understand the objectives } \\
\text { the objectives of the experiment. } & \text { and how these are to be achieved. }\end{array}$ \\
\hline
\end{tabular}

2. Collect relevant background information.

3. New step proposed: Select process control strategy

4. Choose response variable(s)

5. Identify control variables, held constant factors and their levels as well as disturbance factors.

6. If possible, specify normal levels, measurement precision, proposed settings or desired levels and related strategies, and predicted effects of control variables and held constant and disturbance factors.

\section{List known and suspected factor} interactions.

8. Choose experimental design and experimental procedure. This includes activities such as choosing sample size and analyzing preexperimental power.

\section{Consider restrictions and} anticipated difficulties in running the experiment.

10. Outline the analysis techniques.

\section{Assign responsibility for} coordination of the experiment. 12. Perform a trial run or pilot experiment if possible.

13. Review and revise the above steps if necessary.
- Process control during experimentation might be unavoidable and if needed it will affect decisions in subsequent steps.

- If process control is exposed to subjective decisions, a pre-established control strategy is crucial.

- Time-delay may be present in variables used as basis for control decisions as well as for variables used to control the process.

- Care is needed when establishing the experimental unit.

- The nature of the process or product often prohibits direct measurements of reactions, and secondary measurements such as pressures or temperatures must be used as proxies for actual process events.

- There are often numerous responses to consider.

- Response variables are typically not independent from one another.

- Calculations (e.g. energy balances) might be necessary to set the levels of the control variables.

- Held constant factors could be affected by the control strategy.

- The flow of raw materials is often an important disturbance factor.

- Working at this detailed level may at certain stages be impractical due to the multitude of responses.

- Forming natural groupings of responses using a-priori information can be helpful during this step.

- Discussing factor interactions and their connection to the abundant responses may be impractical.

- The process typically exhibits inertia between runs before a new steady-state is attained. This affects the length of the runs.

- Steady-state conditions are in practice a matter of definition.

- Split-plot type designs may be necessary, since complete randomization of runs often needs to be abandoned.

- Designs need to be robust to equipment failure, and thereby missing variable measurements or even missing runs.

- Logistics planning and handling can constitute a large portion of the effort when planning and performing experiments.

- Break-down of process equipment is unwelcome but possible and strategies to handle likely failures should be developed.

- Some factor combinations may be anticipated to invoke process difficulties during the experiment. Strategies to handle such difficulties should be developed.

- Logging frequency may differ between all variables of the experiment.

- Time lag may differ between all variables of the experiment.

- Frequent logging of responses produces autocorrelated data.

- A multivariate approach to analysis should be strongly considered.

- Coordination of all involved may be a considerable task.

- Experiments may run around the clock.

- Trial runs are often unrealistic or even impossible. 
Firstly, a structured planning approach is recommended independent of the type of process. Using the planning process presented in Table 2 for planning experiments in a continuous process is recommended after taking into account the considerations discussed in this article. However, we would like to discuss the most important conclusions in this section.

Since process control might be unavoidable during experimentation, control issues require careful consideration during the planning phase, especially if it involves human interventions. A control strategy needs to be developed and it is vital that this strategy, as well as the objective of the experiment, is understood by those exercising the control. The relation between control actions and the experimental results must be transparent.

The experimenter in a continuous process will have to determine the state of the product without pieces that are directly measurable, i.e. the experimental unit of the continuous product flow has to be determined. Measurements taken at pre-established time periods will constitute the experimental unit. Due to the dynamic characteristic of the continuous process, i.e. the process inertia and the time required for each experimental run to attain a steady-state condition, this is sometimes an involved task. In addition, steady-state might be a matter of definition for semi-stable or unstable processes, such as the one discussed in this article.

In continuous processes, response data is often abundant, correlated, generated with different frequency, have different degree of measurement error and often have different degree of autocorrelation. In addition, the time lags between any intervention and a response may differ significantly. The lack of possibilities to directly measure the states of the product, due to its fuzzy state or due to difficult processing environments are often compensated by an abundance of gauges measuring secondary properties such as flow meters, thermocouples, pressure sensors and so forth. The plentitude of secondary variables complicates e.g. predicting effects, estimating measurement precisions and disentangling suspected interactions. We therefore argue that the responses could be grouped together during preexperimental planning according to process experience and a-priori knowledge about correlations. In this way, the groups could be handled in the same way as individual responses and the detail maintained somewhat during the steps of the planning process. Due to the characteristics of response data mentioned above, we argue that a multivariate approach to data analysis is essential.

When using factorial designs in a continuous process, the choice of design must often be able to withstand failures of process equipment, and thereby missing data in one or few of the planned runs. It is important for the engineer to develop strategies to handle such events during pre-experimental planning. Due to process inertia, quite lengthy runs are necessary and this also increases the risk of having to delete the results of a run due to equipment failure or an alarming processing state. Complete randomization of run order is often difficult and therefore split-plot type designs are generally necessary in order to use factorial designs in continuous processes.

As running continuous processes tend to require the involvement of many people, an experimental effort in these processes highlights the importance of effective information and communication in order to ensure that actions taken will have a minimal effect on the experimental purpose. Further, we have found the need for logistics planning to be greater than expected. Because of the large volumes of material handled, logistics planning can make or break the entire effort.

The studied process in this paper is a pilot plant specifically designed for research purposes, i.e. a "production plant for experimental results." Experimenting in continuous processes normally means conducting experiments in a plant designed for full-scale production. The authors are aware of the further practical complications that are implied in such situations. However, we argue that these only strengthen the need for a structured preexperimental planning process. 


\section{ACKNOWLEDGEMENTS}

The authors are grateful to all members of the LKAB "EBF methodology development project" for important contribution to the results presented here. We also gratefully acknowledge the financial support from LKAB that made this research possible. The authors thank the editor and the reviewers for their valuable suggestions for improvement of this article.

\section{ABOUT THE AUTHORS}

Erik Vanhatalo is a doctoral candidate in Quality Technology at the Department of Business Administration and Social Sciences at Luleå University of Technology (Sweden). He received his Master's degree in Industrial Engineering and Management in 2004 and his research is focused around experimental strategies for continuous processes. Since 2004 he has also been teaching Quality Technology and Quality Management at the Faculty of Technology.

Bjarne Bergquist is Associate Professor of Quality Technology at the Department of Business Administration and Social Sciences at Luleå University of Technology. He received his $\mathrm{PhD}$ in Materials Science in 1999 from Linköping University, where he performed experiments on batch and continuous processes in the powder metallurgical industry. He also has a background as process engineer, doing experiments in a paper mill. He teaches Quality Engineering and Quality Management at the Faculty of Technology. His current research interests are Design of Experiments, quality control, quality management and stakeholder theory. He is a member of the European Network for Business and Industrial Statistics (ENBIS).

\section{REFERENCES}

Bergquist, B. (1997). Property Variation in Sintered Steel: Design of Experiments, International Journal of Powder Metallurgy, 33(2):30-40

Bergquist, B. (1999). New Insights Into Influencing Variables of Water Atomization of Iron. Powder Metallurgy, 42(4):331 - 343.

Bergquist, B., Vanhatalo, E. (2007). Contrast Detection using A-Priori Information. Submitted for publication.

Black-Nembhard, H., Valverde-Ventura, R. (2003). Integrating Experimental Design and Statistical Control for Quality Improvement. Journal of Quality Technology, 35(4):406423.

Box, G. E. P. (1957). Evolutionary Operation: A Method for Increasing Industrial Productivity. Applied Statistics, 6(2):81-101.

Coleman, D. E. \& Montgomery, D. C. (1993). A Systematic Approach to Planning for a Designed Industrial Experiment. Technometrics, 35(1):1-12.

Dean, A., Voss, D. (1999). Design and analysis of experiments. New York, Springer.

Dennis, D., Meredith, J. (2000). An Empirical Analysis of Process Industry Transformation Systems. Management Science, 46(8):1085-1099.

Duchesne, C., MacGregor, J. F. (2000). Multivariate Analysis and Optimization of Process Variable Trajectories for Batch Processes. Chemometrics and Intelligent Laboratory Systems, 51:125-137.

Eriksson, L., Johansson, E., Kettaneh-Wold, N., Trygg, J., Wikström, C., Wold, S. (2006). Multi- and Megavariate Data Analysis: Part I Basic Principles and Applications. Umeå, Sweden, Umetrics AB.

Federer, W. T., King, F. (2007). Variations on Split Plot and Split Block Experiment Designs. Hoboken, N.J., Wiley. 
Geerdes, M., Toxopeus, H., van der Vliet, C. (2004). Modern Blast Furnace Ironmaking: An Introduction. Düsseldorf, Germany, Verlag Stahleisen GmbH.

Hahn, G. J., Dershowitz, A. F. (1974). Evolutionary Operation To-day-Some Survey Results and Observations. Applied Statistics, 23(2):214-218.

Hallin, M., Hooey, L., Sterneland, L., Thulin, D. (2002). LKAB's Experimental Blast Furnace and Pellet Development. La Revue de Métallurgie-CIT, 4:311-316.

Hild, C., Sanders, D., Cooper, T. (2000). Six Sigma* on continuous processes: How and Why it Differs. Quality Engineering, 13(1):1-9.

Kourti, T. (2005) Application of Latent Variable Methods to Process Control and Multivariate Statistical Process Control in Industry. International Journal of Adaptive Control and Signal processing, 19:213-246.

Kourti, T., MacGregor, J. F. (1995). Process Analysis, Monitoring and Diagnosis, using Multivariate Projection Methods. Chemometrics and Intelligent Laboratory Systems, 28:3-21.

Lewin, K. (1946). Action Research and Minority Problems. Journal of Social Issues, 2:34-46.

Montgomery, D. C. (2005). Design and analysis of experiments, $6^{\text {th }}$ ed. Hoboken, N.J., Wiley.

Phadke, M. S. (1989). Quality Engineering Using Robust Design. Lindon, Prentice-Hall International.

Saunders, I. W., Eccleston, J. A. (1992). Experimental Design for Continuous Processes. The Australian Journal of Statistics, 34(1):77-89.

Schmidt, S. R., Launsby, R. G. (1994). Understanding industrial designed experiments, $4^{\text {th }}$ ed. Colorado Springs, Colo., Air Academy Press.

Vanhatalo, E., Vännman, K., Hyllander, G. (2007). A Designed Experiment in a Continuous Process, Helsingborg, Sweden: the $10^{\text {th }}$ QMOD conference proceedings.

Wikström, C., Albano, C., Eriksson, L., Fridén, H., Johansson, E. Nordahl, Å., Rännar, S., Sandberg, M., Kettaneh-Wold, N., Wold, S. (1998). Multivariate Process and Quality Monitoring applied to an Electrolysis Process. Part II. Multivariate time-series analysis of lagged latent variables. Chemometrics and Intelligent Laboratory Systems, 42:233-240.

Wormbs, G., Larsson, A., Alm, J., Tunklint-Aspelin, C., Strinning, O., Danielsson, E., Larsson, H. (2004). The use of Design of Experiments and Sensory Analysis as Tools for the Evaluation of Production Methods for Milk. Chemometrics and Intelligent Laboratory Systems, 73:67-71.

Yin, R. K. (1994). Case study research : design and methods, $2^{\text {nd }}$ ed. Thousand Oaks, CA, Sage.

Zuo, G. (2000). Improving the Performance of the Blast Furnace Ironmaking Process. Department of Chemical and Metallurgical Engineering. Luleå, Luleå University of Technology, Sweden, Doctoral Thesis. 